\title{
Spectrum Sensing using Energy Detection Technique for Cognitive Radio Networks using PCA Technique
}

\author{
R. Sindhubargavi", M. Yuvasrri Sindhu and R. Saravanan
}

Sastra University, Thanjavur, Tamilnadu, India; sindhubargavi@gmail.com

\begin{abstract}
With the growth of subscribers in wireless and data communication there is a scarcity of additional bandwidth to meet the demand. For the efficient usage of the spectrum bandwidth we go for a technology which is Cognitive Radio technique. The spectrum holes in the unutilized spectrum are to be used efficiently for the communication for the secondary user. The Cognitive Radio uses spectrum sensing techniques for determining the spectrum holes in the network. In order to improve the efficiency of the energy detection technique it is combined and used with the statistical Principal Component Analysis (PCA) technique. In conventional PCA technique the signal space power to the noise space power does not match with the actual SNR so a PCA correction technique is followed. The correction factor is applied to the noise space power and signal space power to equate it to the actual SNR. The threshold energy required is determined from the modified energy detection technique and the probability of false alarm value. The proposed method is the combination of PCA and energy detection spectrum sensing. The correction factor is applied to the PCA and it clearly defines the process for threshold Energy computation.
\end{abstract}

Keywords: Cognitive Radio, Energy Detection, Principal Component Analysis, Spectrum Sensing

\section{Introduction}

Spectrum scarcity has become one of the main challenging obstacles to the development of new wireless communication technologies. In order to avoid interference, different wireless networks should operate in separate bands. By this approach a greater portion of the spectrum is being left unutilized. DSA technique is used to allocate the spectrum holes or white spaces of the licensed primary users to the unlicensed secondary users. The bandwidth allocation is different for different areas it is based upon the geography of a country. A fixed set of bandwidth is allocated for Military applications which cannot be accessed for commercial use. It is not possible for changing the allocated bandwidth. The spectrum utilization is inefficient even after the effective allocation of spectrum. The Figure 1 shows the occupancy of the spectrum, it is that the spectrum is not efficiently used and there are holes in the spectrum. The under utilization of the spectrum is due to:

1. The occupancy of the spectrum is high during the day time and less during the night time.
2. The presence of guard bands in the spectrum to prevent the adjacent channel interference.

This paper is based on locating the inefficient usage of spectrum.

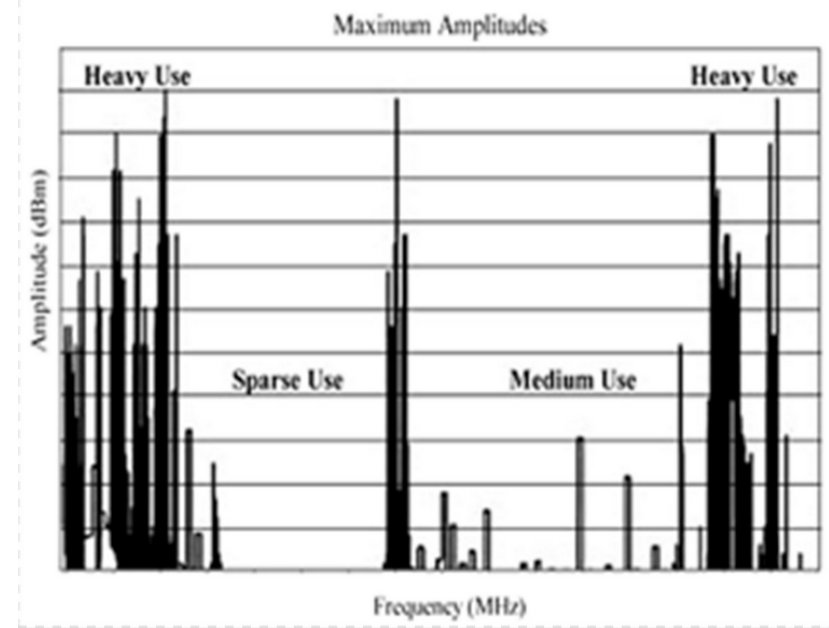

Figure 1. Spectrum Occupancy. 
A Cognitive Radio (CR) is an intelligent wireless communication system, it can obtain information from its neighboring environment and it adjusts its operating parameters thereby increasing its communication channel reliability and accessing dynamically the unused resources leading to a more efficient usage of spectrum. Spectrum Sensing (SS) is one of the common techniques used by a cognitive radio to locate the White Spaces (WS). It just process on the received signal in order to determine the presence of the licensed user in a band. It requires that CRs must be able to detect the signal at very low SNRs in a limited amount of time so that they won't cause any interference to the PUs.

Spectrum sensing has different sensing techniques for detecting the signal: matched filter, energy detector and feature detector ${ }^{6}$. The detection using matched filter is an optional signal detection technique. The demerit of matched filter based detection is that the CRs require a separate receiver for each and every PU. Cyclostationary based feature detection method is more effective method for the detection of very weak signals from noise background. Energy detection is an optional technique that performs non-coherent detection. The drawbacks of energy detection technique is setting up of threshold, detection of spread spectrum signal etc. ${ }^{1}$. The main advantage of energy detection technique is its simplicity in implementation and the short sensing time.

\section{Principle of Energy Detection}

The most extensively used sub-optional signal detection technique in radiometry is the Energy Detection technique. This technique is also used in both time and frequency domain. The target is given to the band pass filter and the power of the signal samples is measured in time domain. Whereas in frequency domain using FFT, the time domain signal is converted to frequency domain signal and the signal power over all frequency band is determined. Figure 2 shows the detection process,

The energy detector baseband equivalent is considered without the loss. The detection is based on the test of these hypotheses:

$$
\begin{aligned}
& \mathrm{H}_{0}: Y[n]=\text { noise } \quad \text { signal is absent } \\
& \mathrm{H}_{1}: Y[n]=\text { signal }+ \text { noise } \quad \text { signal is absent }
\end{aligned}
$$

$n=1 \ldots N$; where $N$ is the observation window

Where $X[n]$, output sample of the signal with $\sigma_{x}^{2}$. The sample noise given by $W[n]$ and it is assumed as Additive White Gaussian noise (AWGN) with mean zero and variance $\sigma_{w}^{2}$. The decision of energy detector is,

$$
T=\sum_{\mathrm{n}=1}^{\mathrm{N}}(Y[n])^{2}
$$

The samples of the signal $X[\mathrm{n}]$ are represented as a function of Gaussian random process with variance $\sigma_{x}^{2}$. Therefore $Y[n]$ is also a Gaussian random process. The number of samples required $N$ is large in the low SNR regime. By central limit theorem, the test data can be approx as a Gaussian distribution.

$$
\begin{aligned}
& \mathrm{H}_{0}: T \sim \operatorname{Normal}\left(N \sigma_{\mathrm{w}}^{2}, 2 N \sigma_{\mathrm{w}}^{4}\right) \\
& \mathrm{H}_{1}: T \sim \operatorname{Normal}\left(N\left(\sigma_{\mathrm{w}}^{2}+{\sigma^{2}}_{\mathrm{x}}\right), 2 N\left(\sigma_{\mathrm{w}}^{2}+\sigma_{{ }_{\mathrm{x}}}^{2}\right)^{2}\right)
\end{aligned}
$$

$P_{d}$ and $P_{f a}$ are the two parameters for performance of detection. Each pair is given with a threshold $\gamma$ value

$\mathrm{T}>\gamma$ signal is present

$\mathrm{T}<\gamma$ signal is absent Then $P_{d}$ and $P_{f a}$ can be calculated as:

$$
\begin{gathered}
p_{d}=p\left(T>\gamma \mid \mathrm{H}_{1}\right)=Q\left(\frac{\gamma-N\left(\sigma_{w}^{2}+\sigma_{x}^{2}\right)}{\sqrt{2 N\left(\sigma_{w}^{2}+\sigma_{x}^{2}\right)^{2}}}\right) \\
p_{f a}=p\left(T>\gamma \mid \mathrm{H}_{0}\right)=Q\left(\frac{\gamma-N \sigma_{w}^{2}}{\sqrt{2 N \sigma_{w}^{4}}}\right)
\end{gathered}
$$

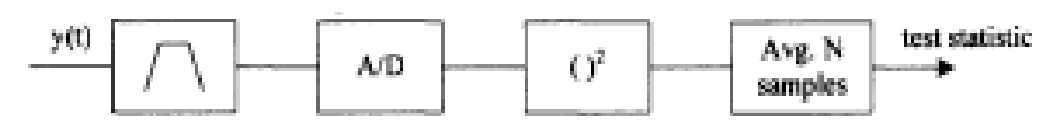

(a) Implementation in time domain

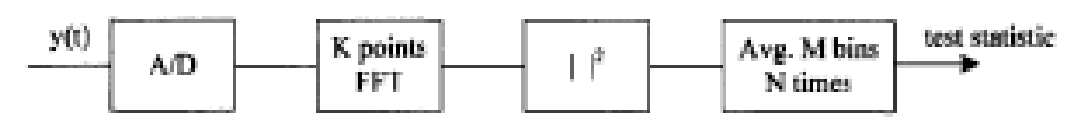

(b) Implementation in frequetsy domain

Figure 2. The process of Energy Detection. 
Where

$$
Q(x)=\frac{1}{\sqrt{2 \pi}} \int_{x}^{\infty} \exp \left(\frac{-y^{2}}{2}\right) d y
$$

We can first set the false alarm probability $P_{f a}$ be a specific constant, in case the signal power is unknown. The threshold can be determined by equation (5). By substituting the threshold in (4) the detection probability $P_{d}$ can be evaluated for the desired number of samples. We can determine the required minimum observation window $N_{\min }$ which is a function of SNR,

$$
N_{\min }=2\left[\left(Q^{-1}\left(p_{f a}\right)-Q^{-1}\left(p_{d}\right)\right) S N R^{-1}-Q^{-1}\left(p_{d}\right)\right]^{2}
$$

where SNR $=\sigma_{X} / \sigma_{W^{*}}$.

Ideally, if the observation window is without restriction, an energy detector meets required $P_{d}$ and $P_{f a}$ at the same time. If the SNR value is minimum the signal will not be detected.

\section{Existing Technique}

\subsection{Conventional Energy Detection Technique}

The energy detection method ${ }^{2}$ is easy in applying as it do not need the knowledge about the primary signal structure. This method is calculates the input signal energy

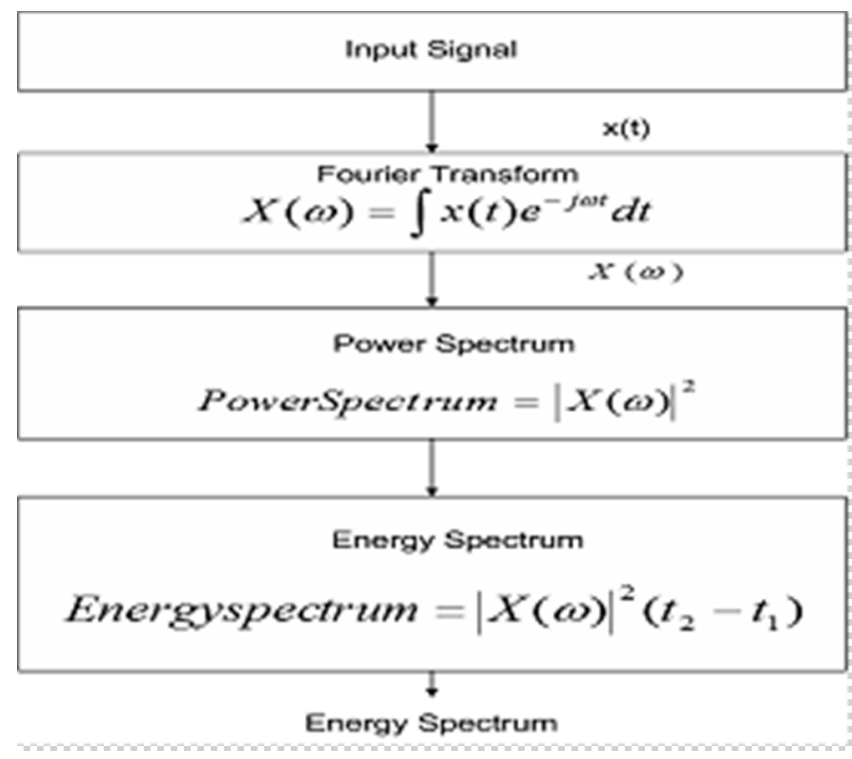

Figure 3. Flow of energy spectrum. and compares it to the threshold energy value. The signals are fixed at a particular frequency when the energy of the signal exceeds the level of threshold. The flowchart of energy detection method is shown in Figure 3.

The Energy detection method is shown in Figure 4 is the conventional energy detection method ${ }^{5}$. The ultimate aim of the work in this paper is to determine the 'hole' at the specific frequency.

\subsection{Principal Component Analysis}

In order to reduce the dimensionality of data ie, to reduce the number of random variables under consideration we go for Principal component Analysis technique ${ }^{6}$. This method is done by analyzing the covariance between the factors. In this method PCA firsts extracts the first component and then it looks through the orthogonal direction of it and so on. This is a standard method for collection of data and for signal compression. Eigen-decomposition method is used to compute the Eigen values of the covariance matrix. Figure 5 shows the separation of Eigen values of the signal and noise.

In order to determine signal power and noise power the following steps should be followed,

1. A signal is first captured and it is grouped into multiple vectors $n$ each of length $M$, it is expressed as, $X$ $(\mathrm{n})=$ input signal of length $\mathrm{n}^{*} \mathrm{M}$

$$
x(n)=\left[x_{1} \ldots x_{\mathrm{M}} x_{\mathrm{M}+1} \ldots x_{2 \mathrm{M}} \ldots x_{\mathrm{Mn}}\right]
$$

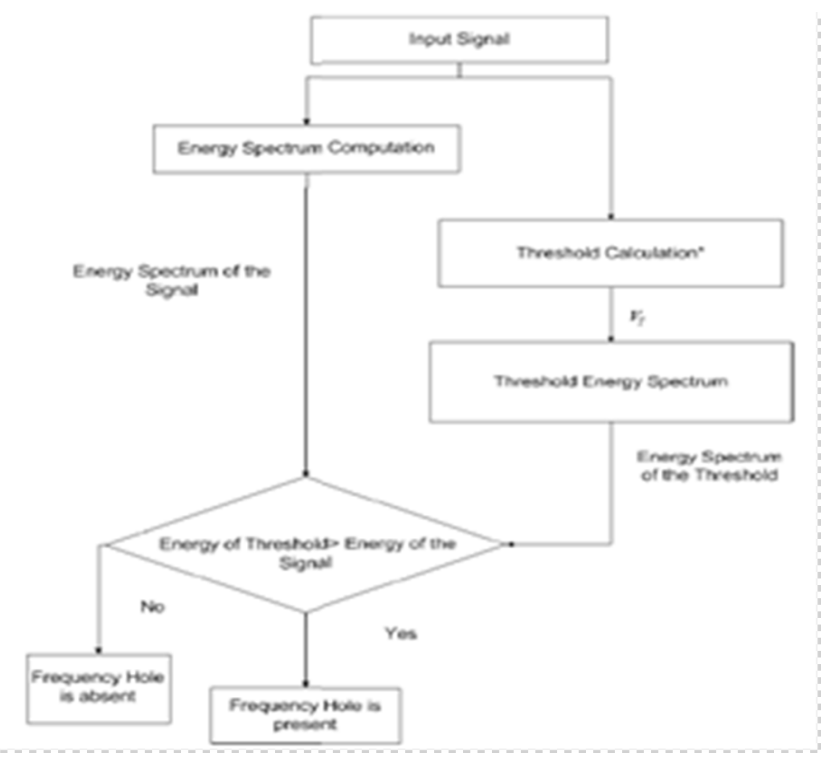

Figure 4. Conventional energy detection technique. 
2. The ' $n$ ' vectors with length $M$ are given by,

$$
\begin{gathered}
\mathrm{L}(1)=\left[x_{1} x_{2} \ldots x_{\mathrm{M}}\right]^{\mathrm{T}} \\
\mathrm{L}(2)=\left[x_{\mathrm{M}+1} x_{\mathrm{M}+2} \ldots x_{2 \mathrm{M}}\right]^{\mathrm{T}}
\end{gathered}
$$

3. Each column vector and its transpose are multiplied to form a matrix $\mathrm{M} \times \mathrm{M}$. The covariance matrix is formed by adding Multiple $\mathrm{LL}_{\text {in }}$ matrices. The size of $\mathrm{LL}_{i}$ matrix is $\mathrm{M} \times \mathrm{M}$.

$$
\mathrm{LL}_{\mathrm{i}}=\mathrm{L}(i) * \mathrm{~L}^{\mathrm{T}}(i)
$$

4. The final covariance matrix is the sum of $\mathrm{LL}_{i}$ matrices. The Eigen values are computed for the final covariance matrix,

$$
\mathrm{Wi}=\sum_{i-1}^{n} \mathrm{LLi}
$$

5. The symmetric matrix $(M \times M)$ is found from the autocorrelation of the input signal.

6. The Eigen-Decomposition of symmetric matrix $(\mathrm{M} \times \mathrm{M})$ results in ' $\mathrm{M}$ ' Eigen values which are arranged in a descending order.

7. The division of Eigen values of the signal and noise is based on 'P' complex exponentials in the input signal.

8. The power of the signal and the noise is determined by the average of the noise Eigen values,

$$
\begin{gathered}
\text { Signal power }==\frac{1}{p} \sum_{i-1}^{p} \lambda \mathrm{i} \\
\text { Noise power }==\frac{1}{M-P} \sum_{i-P-1}^{M} \lambda \mathrm{i}
\end{gathered}
$$

The signal power increases with the increase in noise power and the ratio of the signal power to the noise power is not equal to the SNR value of the system. So the output

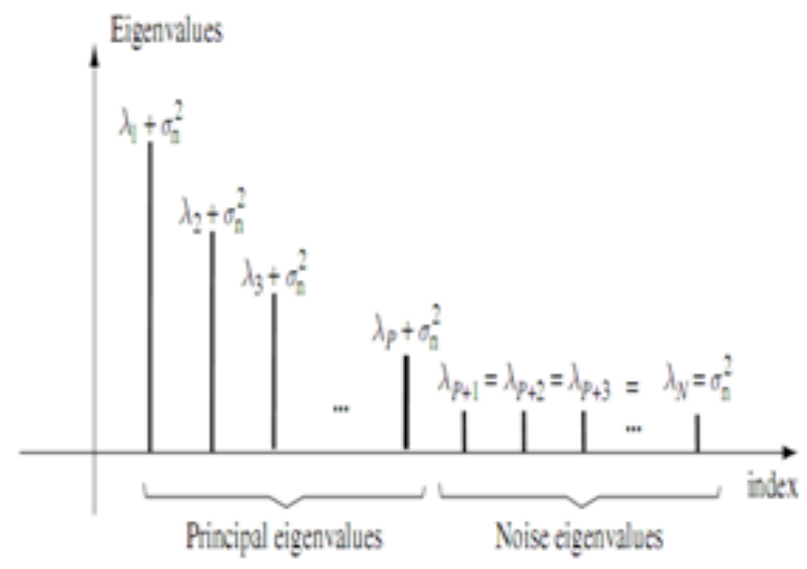

Figure 5. Eigen values of the covariance matrix. of the conventional PCA technique needs to be corrected (Figures 6 and 7).

\section{Proposed Technique}

\subsection{Correction to the PCA Technique}

(Figures 8 and 9)

1. The autocorrelation of the input signal is obtained which results in the symmetric covariance matrix $(\mathrm{M} \times \mathrm{M})$.

2. 'M' Eigen values are arranged in descending order and it is obtained by the Eigen decomposition of the $\mathrm{M} \times \mathrm{M}$ matrix.

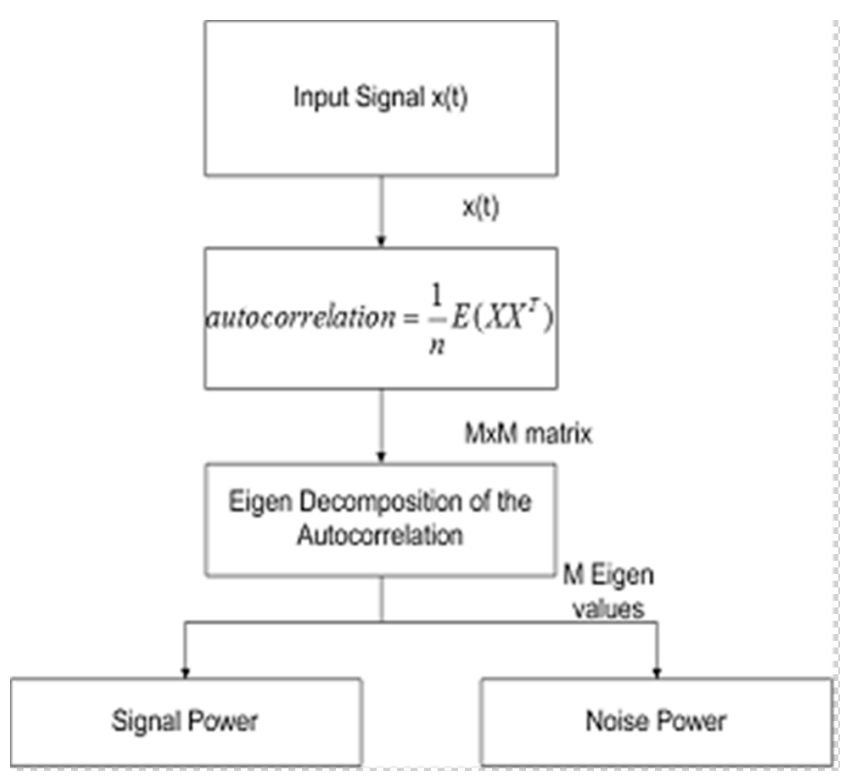

Figure 6. Conventional PCA technique PCA.

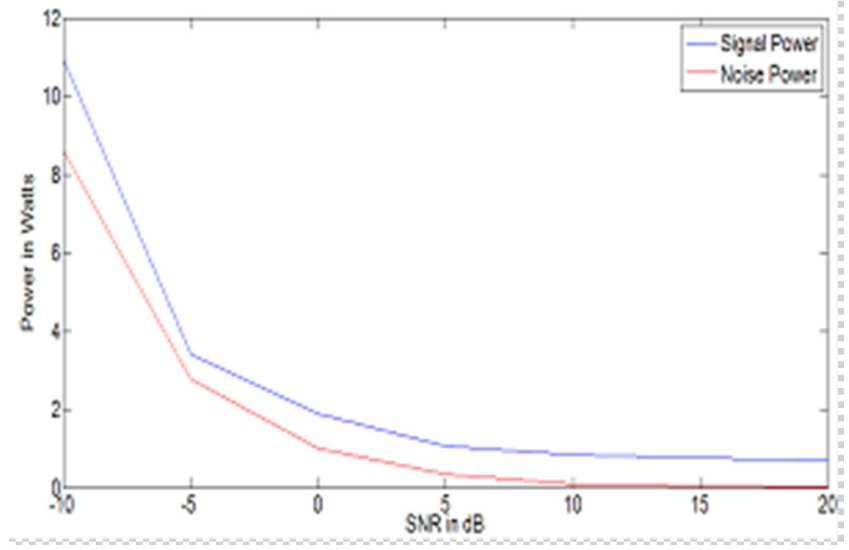

Figure 7. Output of the conventional. 


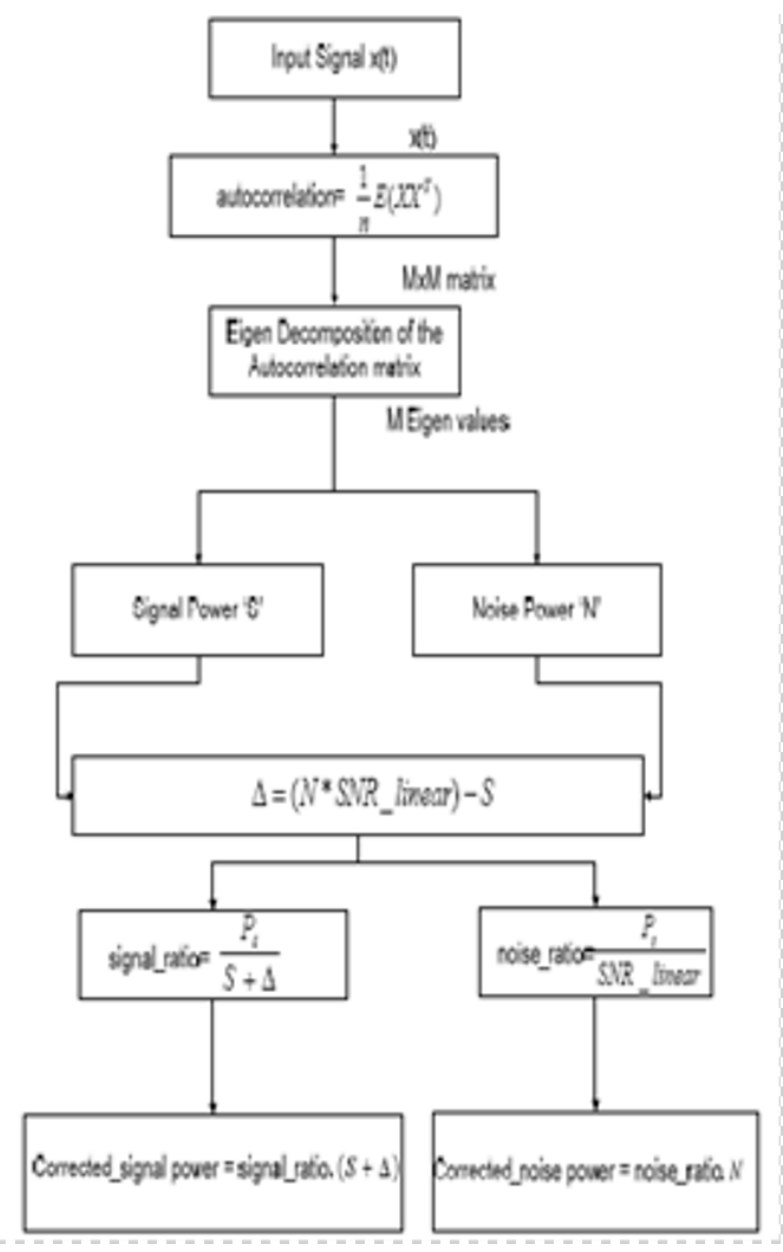

Figure 8. Correction to the PCA technique.

3. The division of Eigen values of the signal and noise is based on ' $\mathrm{P}$ ' complex exponentials in the input signal.

4. The ratio of the signal ' $\mathrm{S}$ ' to the noise ' $\mathrm{N}$ ' determined from the conventional PCA technique do not match the SNR of the input signal hence, ' $\Delta$ ' is added to the signal power 'S'. So the ratio of 'S $+\Delta$ ' to the noise is equated to the SNR of the input signal. ' $\Delta$ ' is calculated as,

$$
\begin{gathered}
\frac{\mathrm{S}+\Delta}{\mathrm{N}}=\mathrm{SNR} \text { linear } \\
\Delta=(\mathrm{N} * \mathrm{SNR} \text { linear })-\mathrm{S}
\end{gathered}
$$

5. The corrected signal power is given by

$$
\text { signal_ratio }=\frac{p i}{s+\Delta}
$$

Corrected_signal power $=$ signal_ratio $(S+\Delta)$

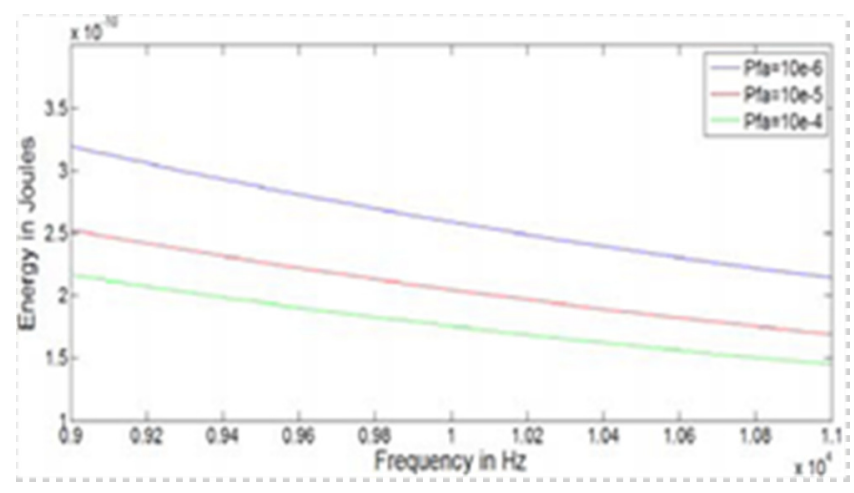

Figure 9. Output of the corrected PCA.

6. The corrected noise power is given by,

$$
\begin{gathered}
\text { noise_ratio }=\frac{p i}{S N R \text { linear }} \\
\text { Corrected_noise power }=\text { noise_ratio.N }
\end{gathered}
$$

The noise power obtained is inserted in eqn (2) to obtain the threshold voltage. The spectra of signal energy is given by,

$$
\left|\mathrm{X}_{\text {in }}(\omega)\right|^{2}\left(\mathrm{t}_{2}-\mathrm{t}_{1}\right)
$$

The spectra of threshold energy is given by,

$$
\left|\mathrm{V}_{\mathrm{T}}(\omega)\right|^{2}\left(\mathrm{t}_{2}-\mathrm{t}_{1}\right)
$$

\subsection{Determination of Threshold Spectrum}

The threshold spectrum is found to be decreasing as the threshold energy increases and also the threshold spectrum is found to be high at a low $\mathrm{P}_{\mathrm{fa}}$ and it is low at high $\mathrm{P}_{\mathrm{fa}}$ Figure 10). The threshold energy spectrum is high at low SNR and the energy spectrum is low at high SNR (Figure 11).

\subsection{Modified Energy Detection Technique}

The threshold voltage computation requires noise power which is determined by the corrected PCA method. Figure 12 shows the flowchart of the modified energy detection technique. Figure 13 is the location of spectrum holes.

The signal power and noise power is estimated using PCA technique and a correction factor is added to the arrived noise. The spectrum hole for the input signal is shown as

\section{Conclusion}

The modified energy detection technique computes the accurate value of threshold voltage and it is determined with the use of accurate noise power determined by 


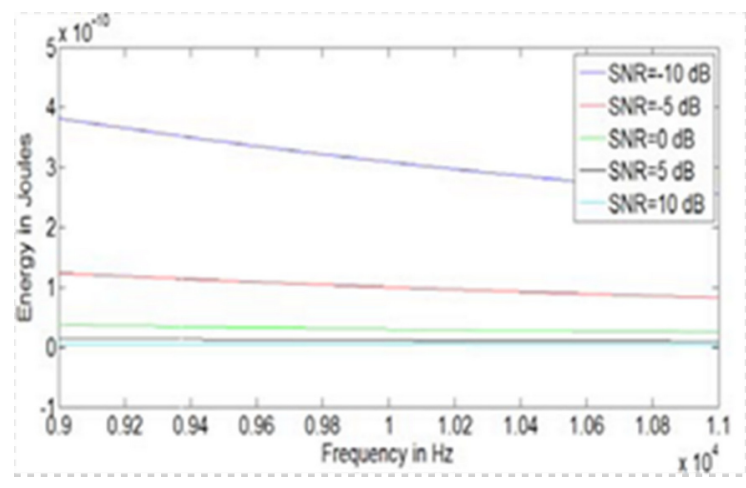

Figure 10. Threshold energy spectrum for SNR values.

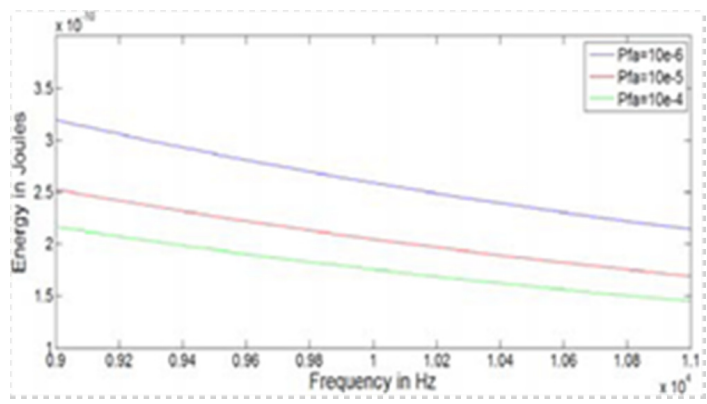

Figure 11. Spectrum of threshold energy for Pfa different.

this proposed method. The energy detection technique compares the threshold energy with the energy of the signal. The spectrum hole is formed if the threshold energy is greater than the energy of the signal. Some conclusions are:

1. The threshold energy is defined from the available noise value, false alarm probability and the frequency.

2. Computation of noise power and signal power is done using PCA detection technique.

3. The ratio of SNR of the conventional PCA technique does not match the actual SNR. The signal power determined by the PCA technique is not equal to the transmitted power.

4. The first order correction term has been applied to the results of the conventional PCA technique and equates it to the ratio of signal power to noise power with the SNR of the actual signal.

\section{References}

1. Liang Y-C, Zeng Y, Peh E, Hoang A. Sensing-throughput tradeoff for cognitive radio networks. IEEE Trans. Wireless Commun. 2008 Apr; 7(4):1326-37.

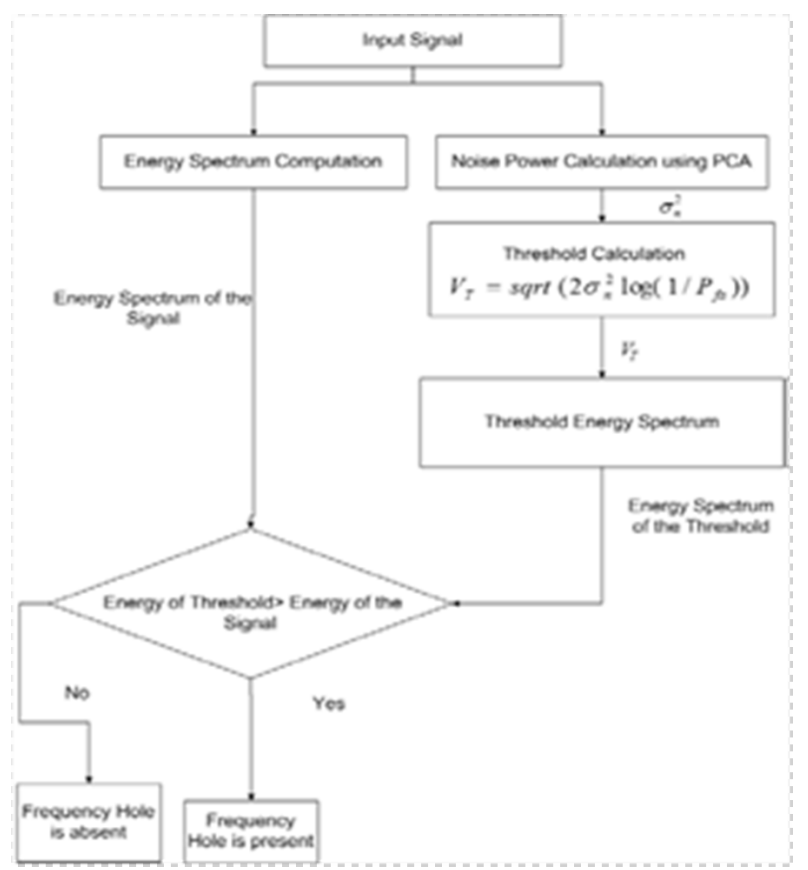

Figure 12. Modified energy detection technique.

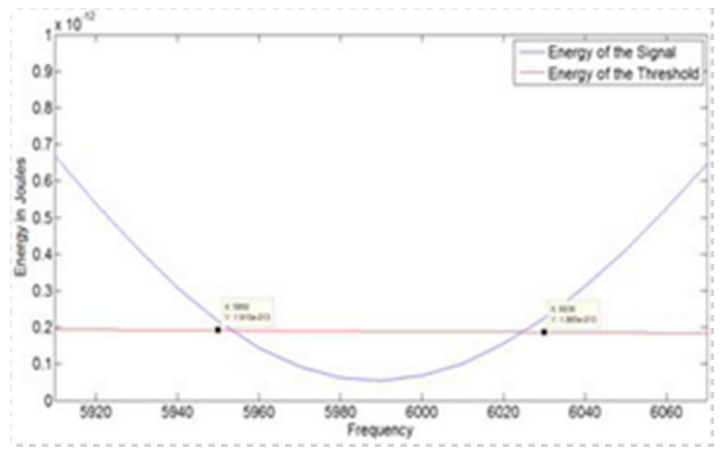

Figure 13. Location of spectrum holes.

2. Kostylev VI. Energy detection of a signal with random amplitude. IEEE International Conference on Communications. ICC; 2002. IEEE. 1606-10.

3. Haykin S, Thomson DJ, Reed JH. Spectrum sensing for cognitive radio. Proc IEEE. 2009 May; 97(5):849-77.

4. Ma L, Li Y, Demir A."Matched filtering assisted energy detection for sensing weak primary user signals. IEEE International Conference on Acoustics, Speech and Signal Processing(ICASSP); 2012. p. 3149-52.

5. Kapoor S, Singh G. Non co-operative sensing: A hybrid model approach. IEEE International Conference on Device and Communication (ICDeCom). 2011. p. 1-5.

6. Maleki S, Pandharipande A, Leus G. Two-stage spectrum sensing for cognitive radios. IEEE International Conference on Acoustics, Speech and Signal Processing (ICASSP); 2010. p. 2946-49. 